EPV026/\#548 SEXUALITY AFTER BREAST CANCER SURGERY IN POSTMENOPAUSAL WOMEN

O Kaabia*, R Bouchahda, S Hidar, M Bibi. Université de Sousse, Faculté de Médecine de Sousse, Gynecology and Obstetrics, Sousse, Tunisia

\subsection{6/ijgc-2021-IGCS.93}

Objectives The main objective of this study was to evaluate the sexual function in married menopausal women after surgery for non metastatic breast cancer

Methods This is a prospective cohort-type study of 200 menopausal women diagnosed then operated on for breast carcinoma between January 2018 and March 2020. Patients were randomized after a multidisciplinary consultation in 2 groups: G1 with patients who had conservative breast surgery and G2 with those who had a mastectomy. Patients with immediate or delayed postoperative breast reconstruction were excluded. The data collection was done in an individual interview, in which 4 validated standardized psychometric assessment scales were used: The Arab Female Sexual Function Index (ArFSFI) for evaluation of sexual function The Locke and Wallace Marital Adjustment Test (MAT) for Assessment of Spousal Agreement The Hospital Anxiety and Depression Scale (HAD-S) for Assessment of Anxiety \& Depression The Body-Esteem Scale for Adolescents and Adults (BESAA) for the evaluation of the body image

Results The two groups were comparable in terms of age and socio-economical characteristics of the patients and their spouses. The mean total FSFI scores were comparable (22 in G1vs 24.5 in $\mathrm{G} 2, \mathrm{p}=0.084$. There was a positive correlation between the husbands' education level and the feminine sexual function $(\mathrm{p}=0.042)$ and between marital agreement and sexual function $(\mathrm{p}=0.004)$.

Conclusions The technique of breast surgery for breast cancer does not influence the sexual function in menopausal women.

\section{EPV027/\#553 CONCORDANCE IN MOLECULAR PROFILES OF INVASIVE BREAST CANCER BETWEEN CORE NEEDLE BIOPSY AND DEFINITIVE OPERATIVE SPECIMEN ANALYSIS}

${ }^{1} \mathrm{O}$ Kaabia*, ${ }^{1} \mathrm{R}$ Bouchahda, ${ }^{1} \mathrm{~S}$ Hidar, ${ }^{1} \mathrm{M}$ Bibi, ${ }^{2} \mathrm{M}$ Mokni. ${ }^{1}$ Université de Sousse, Faculté de Médecine de Sousse, Gynecology and Obstetrics, Sousse, Tunisia; ${ }^{2}$ Université de Sousse, Faculté de Médecine de Sousse, Pathology and Cytology, Sousse, Tunisia

\subsection{6/ijgc-2021-IGCS.94}

Objectives The core needle biopsy (CNB) is an attractive alternative to surgical biopsy for the purpose of characterizing completely a malignant breast lesion for a tailored management. The purpose of this work is to study the concordance of the molecular profile of invasive breast cancer between the $\mathrm{CNB}$ and definitive pathology examination.

Methods We conducted a case-control study where each subject was her own control, including all patients with primary malignant tumors of the breast, collected prospectively, in our Department of Pathology and Cytology and treated at the Department of Gynecology and Obstetrics of the same hospital from January 1, 2015, to July 31, 2017. The studied molecular profile parameters were estrogen receptors (ER), progesterone receptors (PR), HER2 receptors (HER2), and Ki67.

Results We included 521 patients. The concordance between $\mathrm{CNB}$ and definitive postoperative specimen analysis with regard to the molecular profile parameters in invasive breast cancer was respectively of $100 \%$ and $96.3 \%$ for ER and PR, with an excellent agreement (respectively, $\mathrm{k}=1$ and $\mathrm{k}=0.905$ ). The agreement in the diagnosis of tumors HER 2 overexpression was strong $(k=0.679)$. There was a difference between $\mathrm{Ki} 67$ tumoral status (cut off at 20\%) in CNB versus definitive postoperative specimen analysis in $53.1 \%$ of the cases with a weak agreement $(k=0.193)$. Consistency between $\mathrm{CNB}$ and postoperative specimen analysis in the distinction of luminal A tumors was $72.8 \%, 66.7 \%$ for luminal B, 90.1\% for Her2 type and $86.4 \%$ for the basal type.

Conclusions CNB was reliable in determining the hormonal receptors' status and the HER2 negative invasive breast cancer.

\section{EPV028/\#556 EVALUATION OF THE EXTEMPORANEOUS PATHOLOGICAL EXAMINATION OF AXILLARY SENTINEL LYMPH NODE DETECTED WITH BLUE DYE}

${ }^{1} \mathrm{O}$ Kaabia*, ${ }^{2} \mathrm{~N}$ Abdessayed, ${ }^{1} \mathrm{R}$ Bouchahda, ${ }^{1} \mathrm{~S}$ Hidar, ${ }^{2} \mathrm{M}$ Mokni, ${ }^{1} \mathrm{M}$ Bibi. ' Université de Sousse, Faculté de Médecine de Sousse, Gynecology and Obstetrics, Sousse, Tunisia; ${ }^{2}$ Université de Sousse, Faculté de Médecine de Sousse, Pathology and Cytology, Sousse, Tunisia

10.1136/ijgc-2021-IGCS.95

Objectives The evolution of the practice and recommendations regarding the axillary lymph node exploration in breast cancer patients tends toward promoting the sentinel lymph node as a gold standard in clinically No patients. This study aims to evaluate the accuracy and conformity of the extemporaneous pathological examination (EPE) with the definitive pathology examination (DPE) of the sentinel lymph node biopsy (SLNB) detected only with blue dye.

Methods We did a retrospective study including all the earlystage breast cancer patients (cT1/2N0) who underwent an axillary SLNB procedure with blue dye in our department of gynecology and obstetrics from 2008 to 2017. We did evaluate the performances of the EPE of the axillary sentinel lymph node by calculating the sensitivity, specificity, false positive, false negative, positive predictive value, negative predictive value, diagnostic efficacy, and the Youden index.

Results We have registered 441 procedures of EPE of axillary SLNB. When confronting the EPE response to the final response, we found that the sensitivity was $90.72 \%$, the specificity $100 \%$. There were no false-positive and $3.30 \%$ of false negatives. The positive predictive value was $100 \%$ and the negative predictive value $95.10 \%$. The diagnostic efficacy of the EPE was $96.46 \%$ and the Youden index 0.91.

Conclusions The EPE is a good tool to evaluate blue dyedetected axillary sentinel lymph nodes during the surgery for early breast cancer.

\section{EPV029/\#569 PREDICTIVE FACTORS OF TOTAL RESPONSE TO NEOADJUVANT CHEMOTHERAPY (NAT) IN BREAST CANCER PATIENTS: A RESTROSPECTIVE OBSERVATIONAL STUDY}

${ }^{1}$ M Bouhani*, 'S Sakhri, 'S Kammoun, ${ }^{2}$ Bettaieb, ${ }^{1}$ A Goucha, ${ }^{1} \mathrm{R}$ Chargui, ${ }^{1} \mathrm{M}$ Driss. ${ }^{1}$ Salah Azaiez Institute of Oncology, Surgical Oncology, Tunis, Tunisia; ${ }^{2}$ Salah Azaiez Institute of Oncology, Surgical Oncology, Tunis, Tunisia

10.1136/ijgc-2021-IGCS.96 
Objectives This is a observational study with the aim to evaluate predictive factors of response to NAT in patients with breast cancer.

Methods It is a retrospective study included 21 patients $t$ who received neaoadjuvant chemotherapy between 2015 and 2019 at salah azaiez institute Tunisia., we collected, socociodemographics (age, gender, and marital status); tumor: localization, staging.

Results Our study included 21 patients who achieved breast pCR, between 2015 and 2019, 21 patients were included. The median age was 48 years (ranging 30-68). All patients had breast cancer and received neoadjuvant chemotherapy. Two patients were diabetic, 3 patients had high blood pressure and 2 had dyslipidemia. The majority of the tumor had a hign grad nuclear (14). The majority of molecular profile was tripe negative (6cases). The evaluation of the response based on imaging firstly and histological examination. Clinically all patient had a complete response. 17 patients underwent radical surgery and 4 conservative surgery. The evaluation of these patients showed that 16 of theme developed recurrence. We concluded that age, nuclear grad, histological type did not effected the response of chemotherapy but this results is insufficient because of the shortage of the serie.

Conclusions To date, no tumour biological factor is available for clinical use in the prediction of chemotherapy response in advanced breast cancer other than oestrogen receptor status, which predicts response to hormonal therapy

\section{EPV030/\#572 NUTRITIONAL STATUS AND QUALITY OF LIFE OF BREAST CANCER PATIENTS NEEDING FOR RESPONSE TO NEOADJUVANT CHEMOTHERAPY: ABOUT 19 PATIENTS}

M Bouhani*, S Kammoun, S Sakhri, H Bouaziz, I Bettaieb, A Goucha, R Chargui, M Driss. Salah Azaiez Institute of Oncology, Surgical Oncology, Tunis, Tunisia

\subsection{6/ijgc-2021-IGCS.97}

Objectives The aims of this study were to report nutritional status in 19 patients with cancer requiring neoadjuvant chemotherapy and to study the influence of nutritional status on their quality of life.

Methods It is a retrospective study included 19 patients $\mathrm{t}$ who received neaoadjuvant chemotherapy between 2015 and 2019 at salah azaiez institute Tunisia., we collected the nutritional status (weight, anorexia grading, type of diet),socociodemographics (age, gender, and marital status); tumor: localization, staging; health status (performance status according to WHO classification, usual weight and body mass index (BMI).

Results Between 2015 and 2019, 19 patients were included. The median age was 52 years (ranging 30-72). All patients had breast cancer and received neoadjuvant chemotherapy. 13 patients were menopaused, two patients were diabetic, 14 patients had high blood pressure and 4 had dyslipidemia. BMI was normal in 6 cases, overweight in 4 cases and obesity in 9 cases. The majority of the tumor were classified T4B. 17 patients had invasive ductal carcinoma. During chemotherapy, 3 patients follow vegetables and fruit diets, 7 follow mixed diets and 9 follow western diet.Three patient had a sport activity, sedentary lifestyle was noted in 9 patients et 7 patients had a normal daily activity. The evaluation of the response based on imaging firstly and histological examination.

Conclusions The nutritional status of patients with cancer requiring neaoadjuvant chemotherapy was relatively preserved. Functional impairment, the presence of anorexia, appear to be independent predictive factors of quality of life in patients who will recivied neaoadjuvant chemotherapy.

\section{EPV031/\#576 PAGET'S DISEASE OF THE NIPPLE: WITH CONCOMITANT BREAST TUMOR: ABOUT 10 CASES}

M Bouhani*, S Sakhri, S Kammoun, H Bouaziz, A Goucha, R Chargui, M Driss. Salah Azaiez Institute of Oncology, Surgical Oncology, Tunis, Tunisia

\subsection{6/ijgc-2021-IGCS.98}

Objectives Paget's disease is an uncommon breast malignancy and often misdiagnosed, it is associated with underlying in situ or invasive breast cancer. The objective of this study is to identify the type of underlying cancer and specify these characteristics.

Methods Nine patients with Paget's disease who were admitted to our hospital were analyzed retrospectively.

Results Our study included nine patients. Six patients were menopaused. Only seven patients presented with clinical findings suggestive of Paget's disease of the breast. The mean size of the tumor was $36 \mathrm{~mm}$ and axillary lymph node were found in seven cases and Mammography and ultrasonography were was performed in all 16 patients and ultrasonography,of the nine mammographic studies, three were negative, in the others cases it showed suspected opacity in three cases, pleomorphic microcalcifications in four cases and both opacity and microcalcifications in three cases.Four patients had multifocality or multicentricity. Modified radical mastectomy was performed in seven patients, mastectomy and sentinel lymph node dissection in two cases in two, and wide local excision with lymph node dissection in one patient. Pathological findings were ductal carcinoma in situ (DCIS) $(n=4)$, invasive ductal carcinoma (IDC) $(\mathrm{n}=1)$, invasive lobular carcinoma $(\mathrm{n}=1)$, DCIS with IDC $(\mathrm{n}=4)$. All tumors had a high nuclear grad.

Conclusions Patients with Paget's disease of the breast have a high incidence of an underlying breast carcinoma. Most of the patients in this study presented late and were more likely to have positive mammograms.

\section{EPV032/\#579 4 BI-RADS MICROCALCIFICATIONS OF THE BREAST: HOW DOES RADIOLOGIC CLASSIFICATION CORRELATE WITH HISTOLOGY?}

M Bouhani*, S Sakhri, F Abidi, S Kammoun, O Jaidane, R Chargui, K Rahal. Salah Azaiez Institute of Oncology, Surgical Oncology, Tunis, Tunisia

10.1136/ijgc-2021-IGCS.99 